Original Paper http://ajol.info/index.php/ijbcs $\quad$ http://indexmedicus.afro.who.int

\title{
Variabilité climatique et rendement maraicher dans les communes de Djougou et de Tanguiéta au Nord-Bénin
}

\author{
Malick BABAH-DAOUDA $^{1 *}$, Afouda Jacob YABI ${ }^{1}$ et Baké OROU WARI ${ }^{2}$ \\ ${ }^{1}$ Laboratoire d'Analyses et de Recherches sur les Dynamiques Economiques et Sociales (LARDES), Université \\ de Parakou, BP 123 Parakou, Bénin. \\ ${ }^{2}$ Laboratoire de Cartographie, (LaCarto) Uiniversité d'Abomey Calavi, 10 BP 1082 Cotonou, Cadjèhoun, \\ Bénin. \\ *Auteur correspondant ; E-mail : babadaoudamalick@yahoo.fr ; Tel : +220 2492517.
}

Received: 27-06-2021 $\quad$ Accepted: 28-09-2021 $\quad$ Published: 30-10-2021

\section{RESUME}

La production maraîchère est potentiellement affectée par les effets de la variabilité des paramètres climatiques en Afrique de l'ouest. C'est pourquoi, la présente étude s'est proposée d'analyser l'impact de la variabilité climatique sur les rendements de la tomate et du piment dans les communes de Djougou et de Tanguiéta. Elle s'est appuyé sur une analyse des tendances pluviométriques et thermométriques de 1971 à 2020 de la zone d'étude et les test statistique de Pettitt (1979) et de corrélation de Spearman. L'évolution annuelle de la pluviométrie moyenne sur la période 1971-2020, a montré une tendance à la hausse dans la zone d'étude. Les années déficitaires représentent $52 \%$ dans la commune de Djougou et $48 \%$ dans la commune de Tanguiéta. Au cours de la période d'étude, la température de la zone d'étude a connu une hausse de $0,39^{\circ} \mathrm{C}$. L'application des tests de stationnarité aux données pluviométriques a permis de déterminer des années de ruptures à Djougou en 1992 et à Tanguiéta à 1989 au seuil de significativité de $95 \%$ synonyme de changements des conditions pluviométriques. Les corrélations établies entre les rendements et les séries pluviométriques ont montré qu'il n'existe pas de relations explicites entre ces deux variables.

(C) 2021 International Formulae Group. All rights reserved.

Mots clés : Variabilité climatique, production agricole, Djougou, Tanguiéta, Bénin.

\section{Climatic variability and vegetables yields in the municipalities of Djougou and Tanguieta in Northern Benin}

\begin{abstract}
Vegetable production is potentially affected by the effects of climate variability in West Africa. This is why, this study proposed to analyze the impact of climate variability on tomatoes and pepper yields in the municipalities of Djougou and Tanguiéta. It has based on an analysis of rainfall and temperature trends from 1971 to 2020 of the region and the statistical tests of Pettitt (1979) and Spearman correlation. The annual evolution of average rainfall over the period 1971-2020 shows an upward trend in the study area. Deficit years represent $52 \%$ for Djougou municipality and $48 \%$ for Tanguiéta municipality. During the study period, the temperature of the study area increased by $0.39^{\circ} \mathrm{C}$. The application of stationarity tests to rainfall data indicated
\end{abstract}


the break in series in 1992 for Djougou municipality and 1989 for Tanguiéta municipality at the 95\% significance threshold synonymous with changes in rainfall conditions. The correlations established between yields and rainfall have shown that there is no explicit relationship between these two variables.

(C) 2021 International Formulae Group. All rights reserved.

Keywords: Climatic variability, agricultural production, Djougou, Tanguiéta, Benin.

\section{INTRODUCTION}

Le climat constitue un facteur qui régit la production agricole et sa variabilité interannuelle conditionne les systèmes socioéconomiques et environnementaux liés à la disponibilité des ressources en eau (Djamanb et al., 2016; Faye, 2017). Ces irrégularités pluviométriques ont pour corollaire la variabilité des dates de démarrage, de fin et de durée des saisons pluvieuses, associées à une plus grande occurrence des déficits hydriques (Noufé et al., 2015). Les pluies représentent l'élément climatique fondamental qui conditionne les différentes activités agricoles, car les fluctuations climatiques entraînent une modification des systèmes culturaux, engendrent un déficit de la balance commerciale, ébranlent le système économique et tout le tissu social (Djohy et al., 2015).

En Afrique en général et en Afrique de l'Ouest en particulier, la variabilité climatique représente une grande menace pour la croissance et le développement durable, ainsi que pour l'atteinte des Objectifs du Développement Durable (SDSN, 2015). La situation synoptique en Afrique de l'Ouest se présente à travers des phénomènes tels que les sécheresses récurrentes, les perturbations des régimes pluviométriques et les déficits pluviométriques de l'ordre de $20 \%$ à $30 \%$ (Noufé et al., 2011). Selon Doukpolo (2014), la sécheresse sévère et généralisée qui a sévi en Afrique de l'Ouest et du Centre au cours des décennies 70 et 80 , représente le plus fort signal climatique observé sur terre en général et en Afrique tropicale en particulier, depuis que des mesures météorologiques sont disponibles. Cette grande période de sécheresse a eu des incidences néfastes non seulement sur les rendements agricoles, mais plus dramatiques sur les conditions d'existence des populations rurales, car le degré de leur vulnérabilité n'a cessé de s'accroître avec les changements climatiques (Sarr, 2006).

$\mathrm{Au}$ Bénin où l'agriculture constitue la base de l'économie avec une contribution de $36 \%$ au Produit Intérieur Brut et de $88 \%$ aux recettes d'exportation (Houssou, 2014), la situation devient de plus en plus préoccupante, surtout dans les communautés rurales où l'essentiel des revenus des ménages provient des activités agricoles. Selon Ogouwale (2006), la péjoration pluviométrique, la réduction de la durée de la saison agricole, la persistance des anomalies, la hausse des températures moyennes, caractérisent désormais les climats du Bénin; Par conséquent, le secteur agricole se trouve confronté à des baisses de rendement de plus en plus remarquables du fait des pratiques culturales utilisées (GIEC, 2007). Dès lors, l'imminence des mesures d'adaptation pour les $61,1 \%$ de la population du pays qui vivent en milieu rural (INSAE, 2003) et dépendant de l'agriculture pour leur subsistance se justifie à plus d'un titre.

La région septentrionale du pays, depuis les années 1958, 1977, 1983 et 2008 subit le plus fort déficit pluviométrique avec une généralisation de la sécheresse et une baisse constante du nombre de jours de pluie dans les différentes stations (Boko et al., 2012 ; Gbassi, 2018). Dans cette région du pays, le maraichage fait partir des activités qui assurent la diversification des habitudes alimentaires et contribuent à la sécurité alimentaire et à la réduction de la pauvreté. Cependant, la productivité maraîchère n'est pas à l'abri de cette dégradation du potentiel hydrique de production, notamment la température, les précipitations, le vent, l'ensoleillement. 
La présente étude a pour objectif d'analyser l'impact de la variabilité climatique sur les rendements de la tomate et du piment dans les communes de Djougou et de Tanguiéta.

\section{MATERIEL ET METHODES \\ Zone d'étude}

Cette étude a été réalisée dans les départements de l'Atacora et de la Donga (Figure 1), correspondant à la zone agroécologique IV (PANA, 2008). Dans le souci de prendre en compte du continuum rural-urbain dans l'analyse, nous avons ciblé deux communes: la commune de Tanguiéta, à caractère rural et frontalière avec le Burkina Fasso et la Commune de Djougou à caractère urbain qui est une ville carrefour. Ces deux communes connaissent un développement de la production maraîchère au cours de ces dernières années.

La commune de Djougou est limitée au nord par les communes de Kouandé et de Péhunco, au sud par la commune de Bassila, à l'est par les communes de Sinendé, de N'dali et de Tchaourou, et à l'ouest par les communes de Ouaké et de Copargo. Le climat de la commune de Djougou est de type soudanoguinéen avec une saison de pluies (avril à octobre) et une saison sèche (octobre à avril).

La commune de Tanguiéta est limitée au Nord par le Parc de la Pendjari, au Sud par les communes de Toucountouna et de Boukombé, à l'Ouest par les Communes de Matéri et de Cobly, à l'Est par les communes de Toucountouna, de Kérou et de Kouandé. Le climat de la commune de Tanguiéta est de type soudano-sahélien avec une saison pluvieuse qui va de Mai à Novembre et une saison sèche qui s'étend de Novembre à Mai.

\section{Cadre théorique}

\section{Relation entre la variabilité climatique et rendements}

Selon Reddy et al. (2000), dans les régions tempérées la variabilité climatique a des effets tantôt positifs, tantôt négatifs sur le rendement. Selon les conclusions contenues dans le Résumé à l'intention des décideurs' du Groupe de travail II (GIEC, 2007) «Les rendements agricoles devraient augmenter légèrement dans les régions de moyenne et haute latitude pour des augmentations moyennes locales de température allant de 1 à $3{ }^{\circ} \mathrm{C}$ selon la culture considérée, et devraient diminuer au-delà dans certaines régions. Aux latitudes plus basses, particulièrement dans les régions ayant des saisons sèches et dans les régions tropicales, les projections montrent des rendements agricoles décroissants, même pour de faibles augmentations locales de température $\left(1\right.$ à $\left.2{ }^{\circ} \mathrm{C}\right)$, ce qui augmenterait les risques de famine. Globalement, le potentiel de production alimentaire devrait croitre avec l'augmentation de température moyenne locale pour une gamme de 1 à $3{ }^{\circ} \mathrm{C}$, mais au-dessus de ces valeurs, il devrait diminuer ». Une hausse de la température augmenterait la cadence d'évaporation, réduisant ainsi le niveau d'humidité disponible pour la croissance des plantes (Atidégla, 2011). Selon les travaux de Ogouwalé (2006), les impacts des péjorations pluviométriques et de la hausse des températures sur les cultures se manifestent fréquemment par les stress hydriques et/ou thermiques. Les travaux de Sossa (2001) ont montré que du fait des variabilités climatiques, les modifications dans le bilan hydrique ne compromettent pas dangereusement le bouclage du cycle du maïs. Mais selon les mêmes auteurs, si le rythme des variations persiste, la production nationale du maïs sera hypothéquée. Selon Noufe et al. (2015), la garantie d'une bonne production est aussi tributaire des conditions liées à une bonne articulation des opérations culturales (défrichement, travail du sol, plantation ou semis, sarclage ou entretien, fertilisation ou non, usages ou non de variétés améliorées, etc.). Ils ont montré à cet effet, que la seule connaissance du régime pluviométrique ne suffit pas à expliquer le rendement d'une culture telle que le maïs pluvial, un rendement faible pouvant aussi bien résulter de conditions hydriques déficitaires qu'excédentaires Noufé et al. (2011). Dans une approche agro 
climatologique proprement dite, ce sont surtout les flux du continuum solplante- atmosphère qu'il convient de renseigner, si l'on veut bien comprendre les interactions complexes entre climat et agriculture (Reyniers et Forest, 1990). Seck et al. (2005) et Salack et al. (2006) ont montré que les rendements agricoles ont connu des déficits drastiques dus aux effets néfastes des changements climatiques actuels et les rendements estimés à l'horizon 2050 connaitront des baisses importantes. Moron (1993) et Morel et al. (2008) ont montré que les précipitations ont diminué de façon relativement brutale vers 1970. Cette baisse a également entraîné celle des productions agricoles qui en dépendent. Selon Sarr (2007) et Salack (2006), les rendements des cultures vivrières (mil, sorgho, maïs, etc.) ont baissé de plus de $60 \%$ à cause des seules variations pluviométriques. Selon Ogouwalé (2006) et Niang (2007) des cultures d'exportation, qui étaient rentables avant 1970, ne le sont plus, car étant inadaptées à la nouvelle situation climatique. C'est le cas du palmier à huile et de la tomate au Bénin méridional ou de l'arachide au Niger.

\section{Mesure de la corrélation pluie et rendement}

Afin de déterminer l'influence des pluies sur l'évolution des rendements, une corrélation est calculée entre les indices climatiques et les rendements au moyen des coefficients de corrélation de Spearman (Spearman, 1904 ; Lehmann et d'abrera 1976 ; Sneyers, 1990). La corrélation de Spearman est l'équivalent non-paramétrique de la corrélation de Pearson. Elle mesure le lien entre deux variables. Si les variables sont ordinales, discrètes ou qu'elles ne suivent pas une loi normale, on utilise la corrélation de Spearman. Cette corrélation n'utilise pas les valeurs des données mais leur Rang. L'interprétation du coefficient de corrélation obtenu reste la même que lorsqu'on utilise une corrélation de Pearson. Le coefficient de corrélation varie entre -1 et $+1,0$ reflétant une relation nulle entre les deux variables, une valeur négative (corrélation négative) signifiant que lorsqu'une des variables augmente, l'autre diminue ; tandis qu'une valeur positive (corrélation positive) indique que les deux variables varient ensemble dans le même sens.

\section{Collecte et analyse des données climatiques}

Les données pluviométriques ont été collectées auprès de l'ASECNA (Agence pour la Sécurité de la Navigation Aérienne en Afrique et à Madagascar), sur la période de 1971 à 2020. Ces données ont permis de déterminer des paramètres de tendance centrale et de dispersion et les indices standardisés et de mettre en évidence les tendances pluviométriques et thermométriques.

\section{Détermination de la durée des saisons culturales}

La saison culturale (SC) ou saison de pluie potentiellement utile est définie comme la suite des décades consécutives pour lesquelles la valeur de la capacité d'évapotranspiration d'un couvert végétal (CEcv en $\mathrm{mm} /$ jours) est significativement supérieure ou égale à $50 \%$ de l'évapotranspiration de référence (ETP) de la période considérée (Eldin, 1989 ; Morel, 2004; Noufe et al., 2011 ; Noufe et al., 2015). Ce seuil est à rapprocher des valeurs du rapport ETM/ETP considérées en début et fin de cycle de culture, et utilisées en tant qu'indices de satisfaction des besoins en eau des cultures en conditions naturelles non irriguées ; ETM est considérée ici comme l'évapotranspiration réelle maximale d'une culture donnée (Noufe et al., 2015).

Ainsi, le besoin en eau optimum d'une culture donnée ou l'évapotranspiration maximale (ETM) s'obtient par le produit de l'ETP et du coefficient cultural (kc) de cette culture; ce qui revient algébriquement à ETM $=\mathrm{Kc} \mathrm{x}$ ETP. Cette valeur a été divisée en trois phases : pré-humide, humide, et post-humide. A partir de ces formules, la pluie $(\mathrm{P})$ a été alors comparée à l'évapotranspiration potentielle (ETP) mensuelle ce qui permet de caractériser les saisons culturales.

Selon Noufe et al. (2015), pour un mois donné, si les pluies (apport d'eau) sont supérieures à l'ETP (la demande en eau), cela voudra dire que les cultures ne manqueront pas d'eau. De la même manière, le rapport ETP/2 
représente un besoin hydrique moyen qui permet à un couvert végétal de démarrer son cycle végétatif et de le terminer dans les conditions optimales. En fin de compte, le bilan climatique calculé traduit le rythme des excédents ou des déficits en eau à l'échelle mensuelle ; car il exprime la différence entre les totaux pluviométriques $(\mathrm{P})$ et la valeur de l'évapotranspiration (ETP), constituant soit le déficit, soit l'excès d'eau disponible pour la recharge de la nappe et pour l'écoulement (Cocheme et Franquin, 1969, cités par Noufe et al., 2015). D'après ces auteurs, la formule du bilan climatique (BC) est la suivante :

$\mathrm{BC}=\mathrm{P}-\mathrm{ETP}$

Avec $: \mathrm{BC}=$ bilan climatique $(\mathrm{mm}) ; \mathrm{P}=$ pluviométrie totale mensuelle $(\mathrm{mm})$; ETP : évapotranspiration potentielle mensuelle (mm).

Ainsi, si :

P-ETP $<0$, BC est déficitaire ;

P- ETP $>0, B C$ est excédentaire ;

$\mathrm{P}-\mathrm{ETP}=0, \mathrm{BC}$ est équilibré.

\section{Indice standardisé des précipitations (ISP)}

La compréhension qu'un déficit pluviométrique a un impact différent sur les eaux souterraines, le stockage des réservoirs, l'humidité du sol et le flux d'écoulement ont conduit au développement de l'indice standardisé des précipitations (ISP) (McKee et al., 1993). L'ISP est un indice simple qui est adopté en 2009 par l'organisation mondiale de la météorologie (OMM) comme un instrument mondial pour mesurer les sécheresses météorologiques (Jouilil et al., 2013, Faye, 2017). Il est exprimé mathématiquement comme suit :

$$
\mathbf{I S P}=\frac{\mathbf{P}_{\mathbf{i}}-\mathbf{P}_{\mathbf{m}}}{\mathbf{S}}
$$

Avec Pi : la pluie du mois ou de l'année i ; Pm : la pluie moyenne de la série sur l'échelle temporelle considérée ; S : l'écart-type de la série sur l'échelle temporelle considérée.

Un événement de sécheresse se produit chaque fois que l'ISP est continuellement négatif et que sa valeur atteint une intensité de -1 ou moins et se termine lorsque l'ISP devient positif.

\section{Indice de température de Lamb}

L'indice de Lamb est utilisé pour l'analyse de la variabilité interannuelle de la température. La même approche méthodologique que celle de l'Indice standardisé des précipitations est utilisée pour l'estimation de cet indice. L'indice de température de Lamb peut être utilisé pour définir la distribution d'années de température normale, froide et chaude. Ainsi, suivant L'HÔTE et al. (2002) l'indice de température de Lamb est donné par la formule :

$$
\mathbf{L}_{\mathbf{i}}=\frac{\mathbf{A}_{\mathbf{i}}-\overline{\mathbf{A}}}{\mathbf{S}}
$$

Avec $\mathbf{A}_{\mathbf{i}}$, la température moyenne pour chaque année, $\overline{\mathrm{A}}$, représente la moyenne annuelle de température obtenue sur l'ensemble de la période de l'étude et $\mathrm{S}$ est l'écart-type estimé sur la période considérée.

Selon cette approche, une année sera considérée comme ayant une saison normale si l'indice de température de Lamb est compris entre $+0,5$ et $-0,5$; comme chaude si l'indice de Lamb est supérieur à 0,5 et enfin comme froide si l'indice de Lamb est inférieur à $-0,5$.

\section{Détection de rupture}

Le logiciel Kronostat 1.01 a été utilisé pour l'analyse des séries chronologiques, en vue de détecter les ruptures liées à une nonstationnarité. Le test de Pettitt (1979) a été retenu pour le test d'homogénéité. Il est d'usage très répandu et est adapté à la détermination d'une rupture unique. Ce test, non paramétrique est dérivé du test de Mann-Whitney. L'absence de rupture dans la série $\mathrm{Xi}$ de taille $\mathrm{N}$ constitue l'hypothèse nulle (Pettitt, 1979).

\section{Collecte et analyse des données agricoles}

Les données sur la production et les superficies de tomate et du piment ont été collectées au niveau du service statistique du Ministère de l'Agriculture, de l'Elevage et de la Pêche (MAEP) et la base de données de la FAO (2020), disponibles en ligne (FAOSTAT). Elles couvrent la période de 2002 à 2020. Les rendements agricoles (quantité produite à l'hectare) sont calculés à partir du rapport entre la production totale et la superficie totale emblavée. 


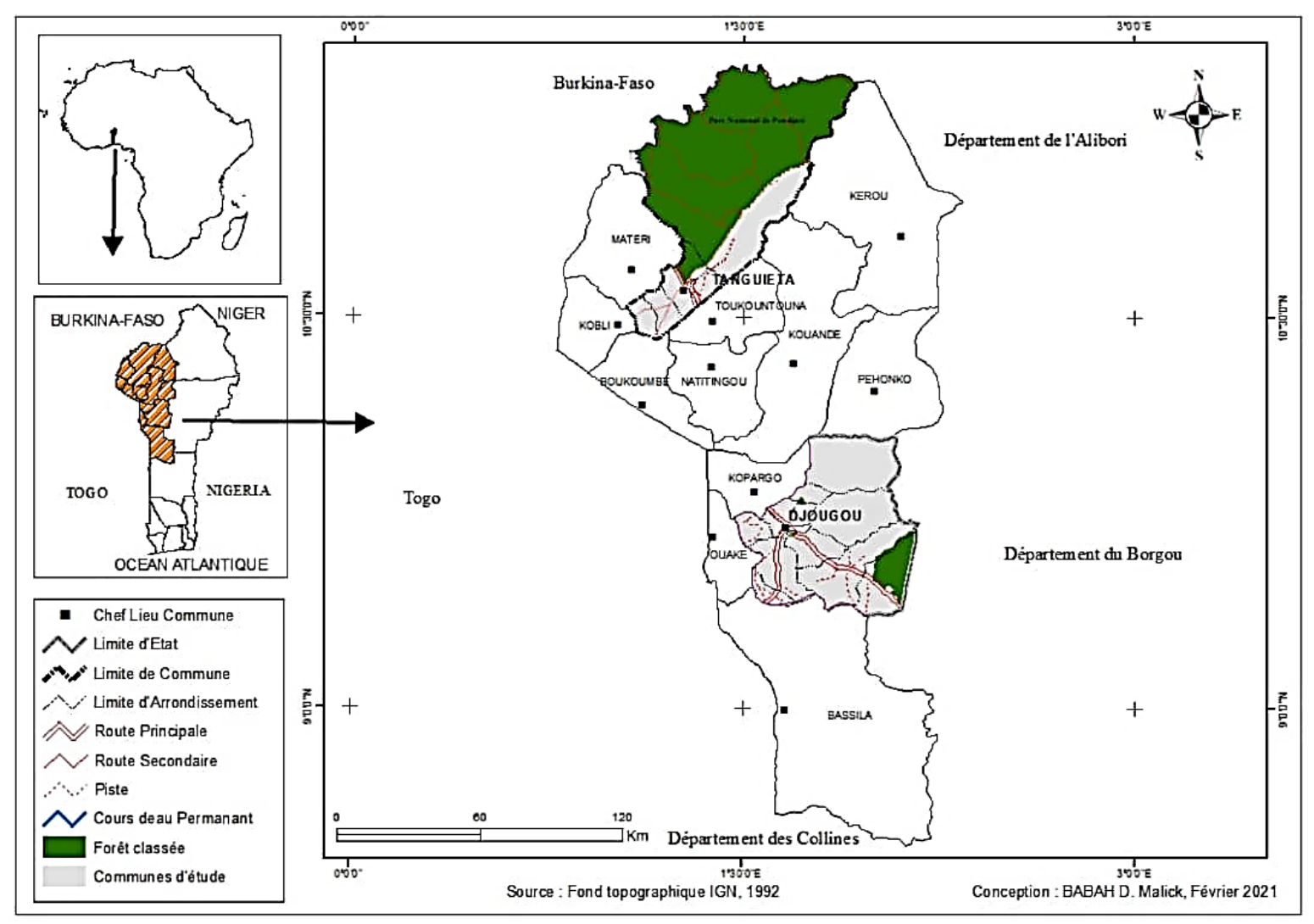

Figure 1 : Localisation de la zone étudiée.

\section{RESULTATS \\ Analyse des régimes pluviométriques mensuelles}

La Figure 2 présente la courbe ombrothermique de 1971 à 2020. Elle indique que le régime pluviométrique de la zone d'étude de 1971 à 2020 est unimodal et est marqué par deux saisons. Dans la commune de Djougou, la saison pluvieuse couvre la période Mai à Novembre et la saison sèche de Novembre à Mai avec un maximum de pluie en Août $(275,5 \mathrm{~mm})$ et le minimum en Janvier (1,0 mm). Tandis que dans la commune de Tanguiéta, la saison pluvieuse couvre la période de Mai à Octobre et la saison sèche de Novembre à Avril avec un maximum de pluie en Septembre $(227,3 \mathrm{~mm})$ et le minimum en Janvier (0,089 mm). La Figure 3 est construite à partir d'une Méthode agro-climatique d'analyse du bilan hydrique développée par
Franquin (1969). Elle montre que la zone D’étude est marquée par trois périodes: la période pré-humide durant laquelle la pluie est globalement inférieure à ETP (Janvier à Mai), la période humide durant laquelle la pluie est globalement supérieure à ETP (Juin à Septembre) et la période post-humide durant laquelle la pluie redevient globalement inférieure à ETP (Octobre à Décembre).

\section{Analyse de la variabilité pluviométrique inter-annuelle}

La Figure 4 présente l'évolution de l'indice Standardisé des Précipitations (ISP) dans les communes de Djougou et de Tanguiéta de 1971 à 2020. L'analyse des précipitations dans la commune de Djougou de 1971 à 2020 montre une tendance à la hausse. Sur les 50 années considérées, les années sèches sont au nombre de 26 , soit $52 \%$ avec des degrés de 
sècheresse variables d'une année à une autre et les années humides sont au nombre de 24 soit $48 \%$ avec des degrés d'humidité variables d'une année à une autre. Dans la commune de Djougou, l'année 1983 a été la plus déficitaire $(-1,25)$ et l'année 2004 la plus humide $(1,17)$. De même dans la commune de Tanguiéta, de 1971 à 2020, on note une tendance à la hausse des précipitations. Sur les 50 années de l'étude, les années sèches au nombre de 24 , soit $48 \%$ et les années humides sont au nombre de 26 , soit $52 \%$. Dans la commune de Tanguiéta, l'année 1989 a été la plus déficitaire $(-1,45)$ et l'année 2009 la plus humide $(1,42)$.

\section{Détection de rupture de stationnarité}

La Figure 5 montre la détection de rupture de stationnarité par le test de Pettitt (1979). Dans la commune de Djougou, l'application du test de Pettit aux séries pluviométriques annuelles de 1971 à 2020 montre une rupture de stationnarité significative au seuil de $95 \%$ au début des années 1992. Il apparait alors deux grandes périodes notamment la période sèche de 1971 à 1992 et la période humide de 1993 à 2020 . La moyenne avant la rupture est de 1199,54 mm et après la rupture est de 1286,92 mm L'écart est de $87,38 \mathrm{~mm}$. Dans la commune de Tanguiéta, le test de Pettit aux séries pluviométriques annuelles de 1971 à 2020, montre une rupture de stationnarité significative au seuil de $95 \%$ au début des années 1989. Deux grandes périodes apparaissent, notamment la période sèche de 1971 à 1989 et la période humide de 1990 à 2020. La moyenne de pluie avant la rupture est de $976,98 \mathrm{~mm}$ et la moyenne après la rupture est de 1111,57 mm, soit un écart de 134,54 mm.

\section{Analyse des tendances thermométriques moyenne de la zone}

La Figure 6 montre la variation de l'indice thermométrique dans le nord-ouest du Bénin de 1971 à 2020. On note une tendance à la hausse des températures moyennes. Sur les 50 années d'études, 22 années ont présenté des températures normales soit $44 \%$; 08 années ont présenté des températures chaudes, soit $16 \%$ et 20 années ont présenté des températures froides, soit $40 \%$ L'évolution de la température moyenne varie entre $26,67^{\circ} \mathrm{C}$ en 1971 à $27,06^{\circ} \mathrm{C}$ en 2020 soit une augmentation de $0,39{ }^{\circ} \mathrm{C}$ La température moyenne la plus élevée a été enregistrée en $2011\left(28,04{ }^{\circ} \mathrm{C}\right)$.

\section{Analyse de la corrélation entre l'évolution des superficies et des productions}

La Figure 7 présente l'évolution des superficies et productions de piment et de tomates de 2002 à 2020 et le Tableau 1 expose les résultats du test de corrélation de Spearman entre les superficies et les productions de la tomate et du piment. De 2002 à 2020, l'évolution des superficies et de la production de la tomate sont fortement corrélées dans les communes de Djougou et de Tanguiéta. Les taux de corrélation de Spearman significatifs à $95 \%$ sont respectivement de 0,935 et 0,875 . Pour la même période, l'évolution des superficies et de la production du piment sont fortement corrélées dans les communes de Djougou et de Tanguiéta. Les taux de corrélation de Spearman significatifs à $95 \%$ sont respectivement de 0,649 et 0,93.

\section{Analyse de l'évolution des rendements et corrélation avec la pluviométrie}

La Figure 8 présente l'évolution des rendements du piment et de la tomate de 2002 à 2020. En superposant la Figure 4 (ISP) à la Figure 8 on constate que la période allant de 2002 à 2020 a été à $47 \%$ humide et $43 \%$ sèche dans la commune de Djougou et à $57 \%$ humide et $43 \%$ sèche dans la commune de Tanguiéta. Ces conditions excédentaires dans la commune de Djougou et de Tanguiéta sont confirmées par le test de Pettitt (Figure 5).

L'analyse de la Figure 8 indique une tendance globale à la hausse des rendements des deux cultures de 2002 à 2020. Cette situation pourrait s'expliquer par les conditions pluviométriques excédentaires au cours de la période. Cependant, les coefficients de corrélation de Spearman établies entre les séries pluviométriques et les rendements (Tableau 2) avec un degré de significativité de 95\% montrent contre toute attente une corrélation assez faible et non significative entre la pluviométrie et les rendements. 


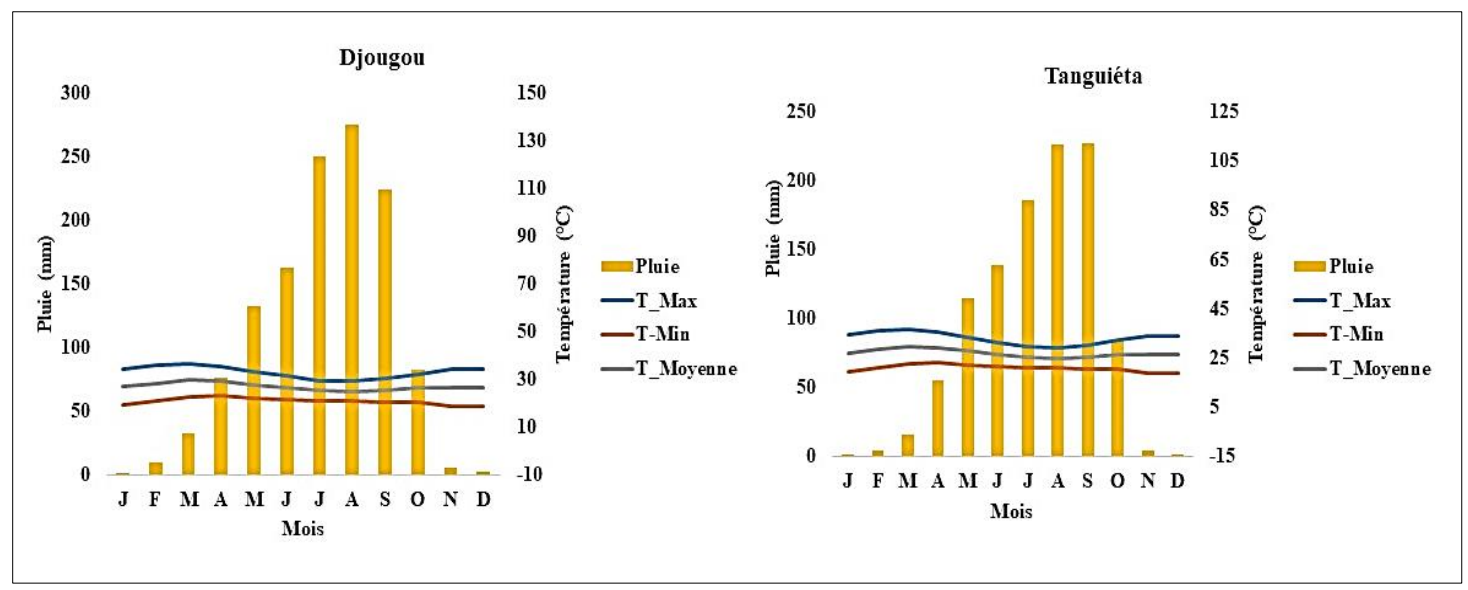

Figure 2 : Courbe ombrothermique de 1971 à 2020.

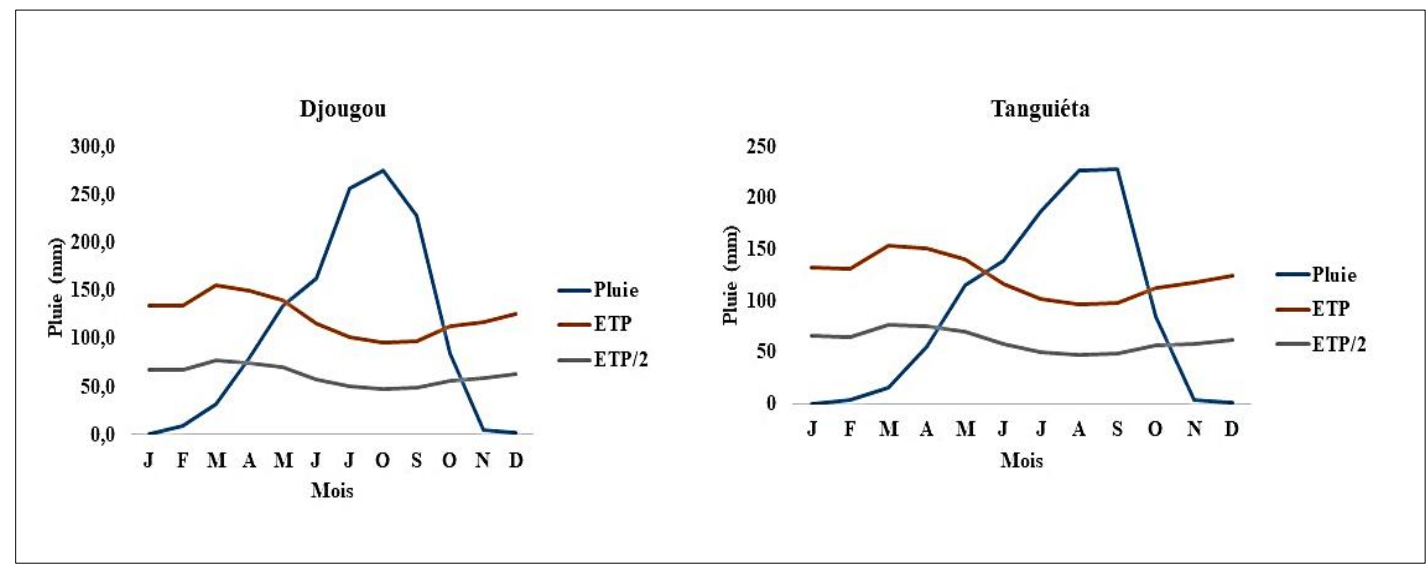

Figure 3 : Courbe de Franquin réalisée à partir des données climatiques recueillies de 1971 à 2020.

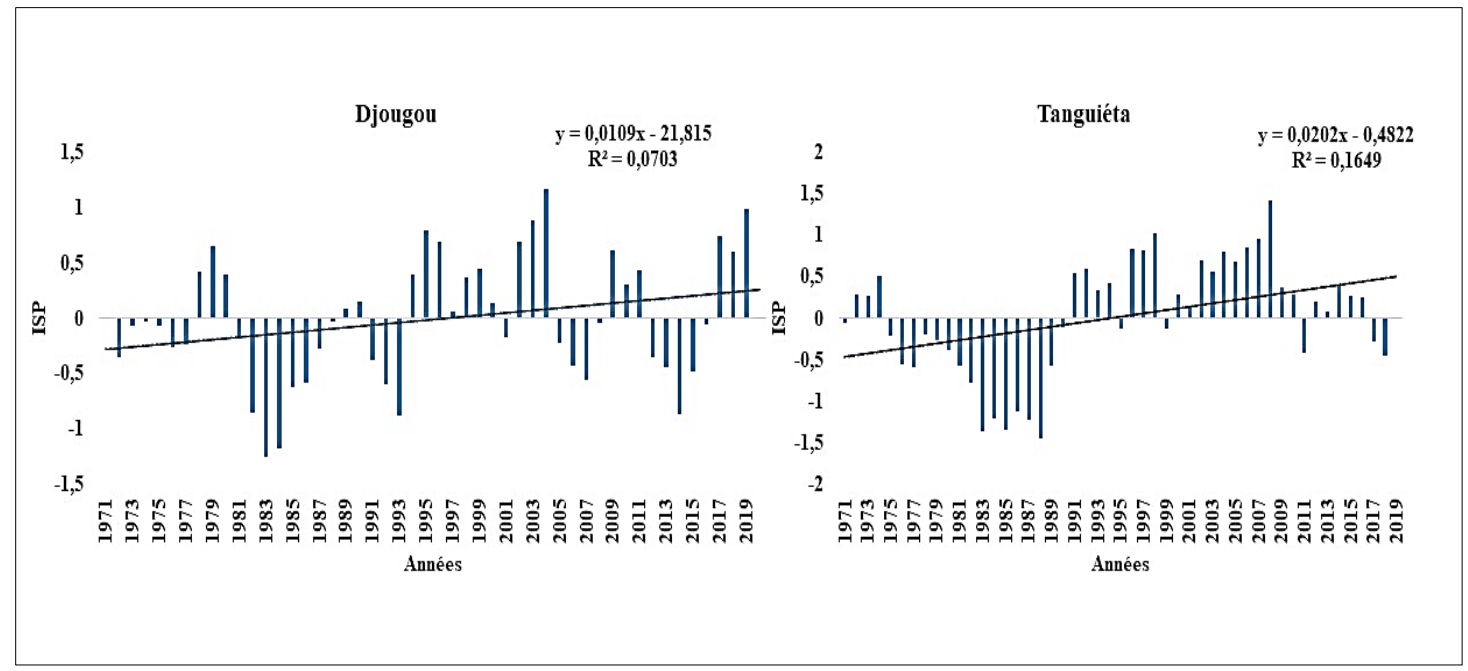

Figure 4 : Indice pluviométrique. 


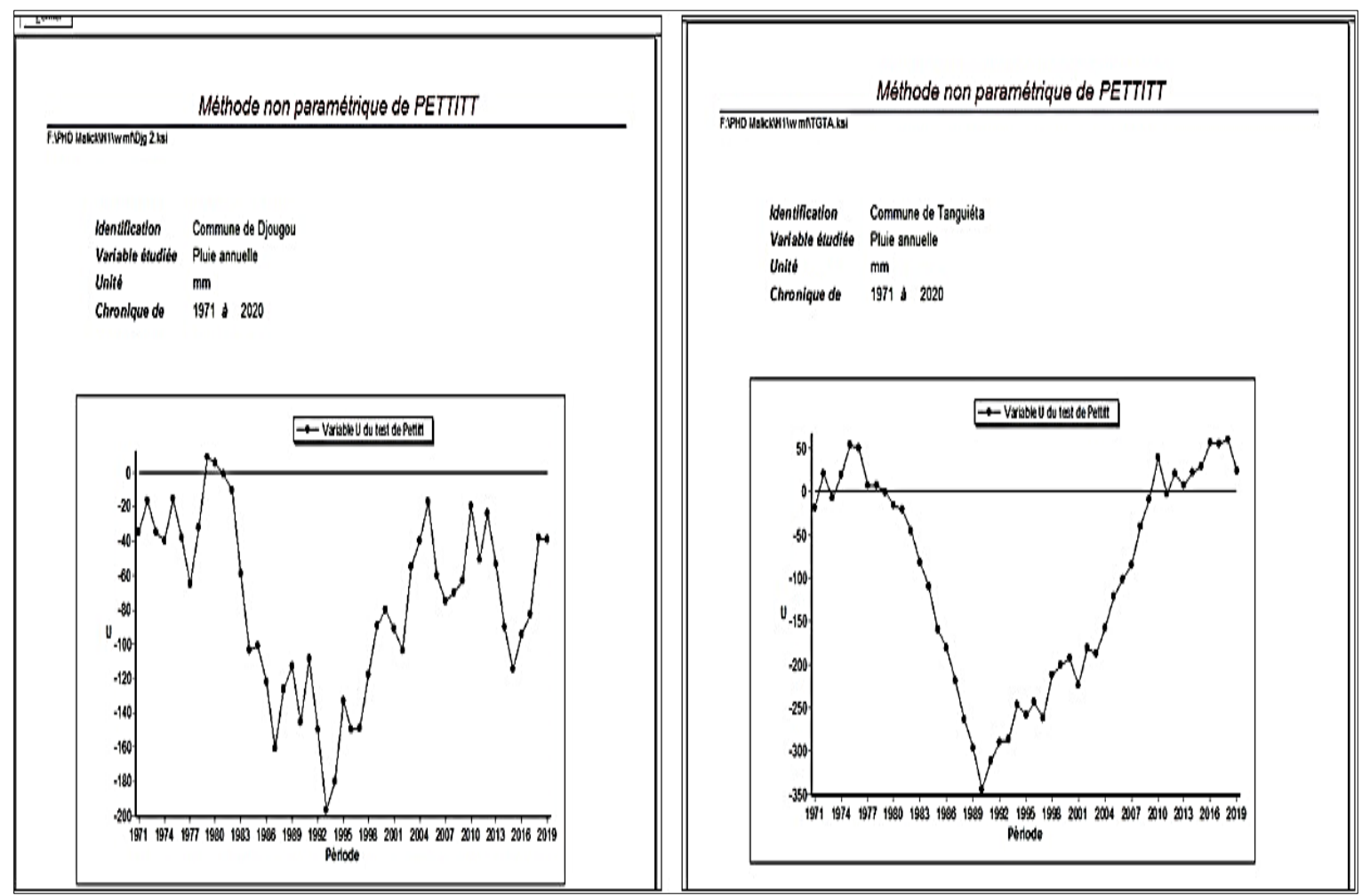

Figure 5 : Rupture de stationnarité par le test de Pettitt.

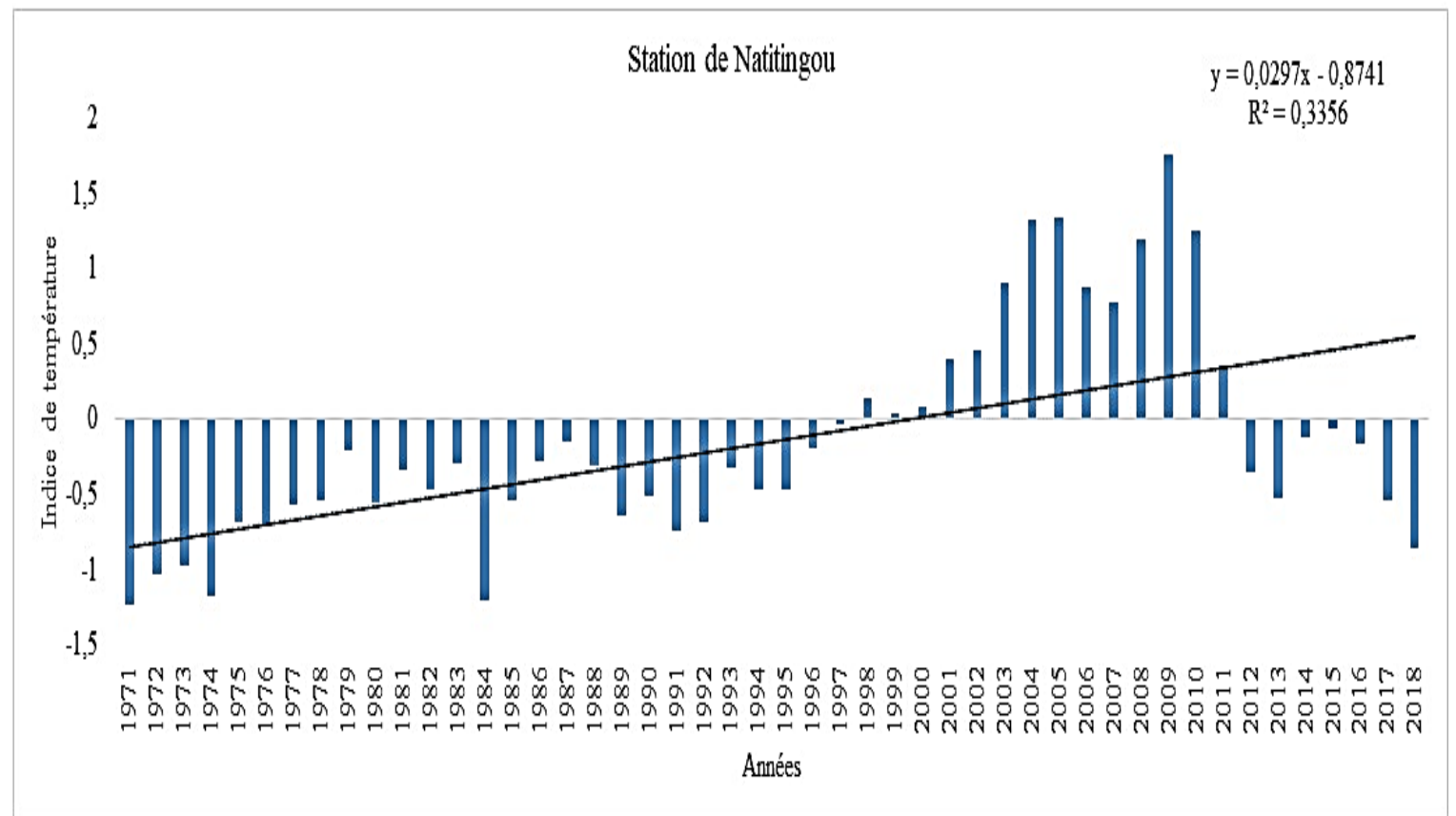

Figure 6 : Indice de température. 


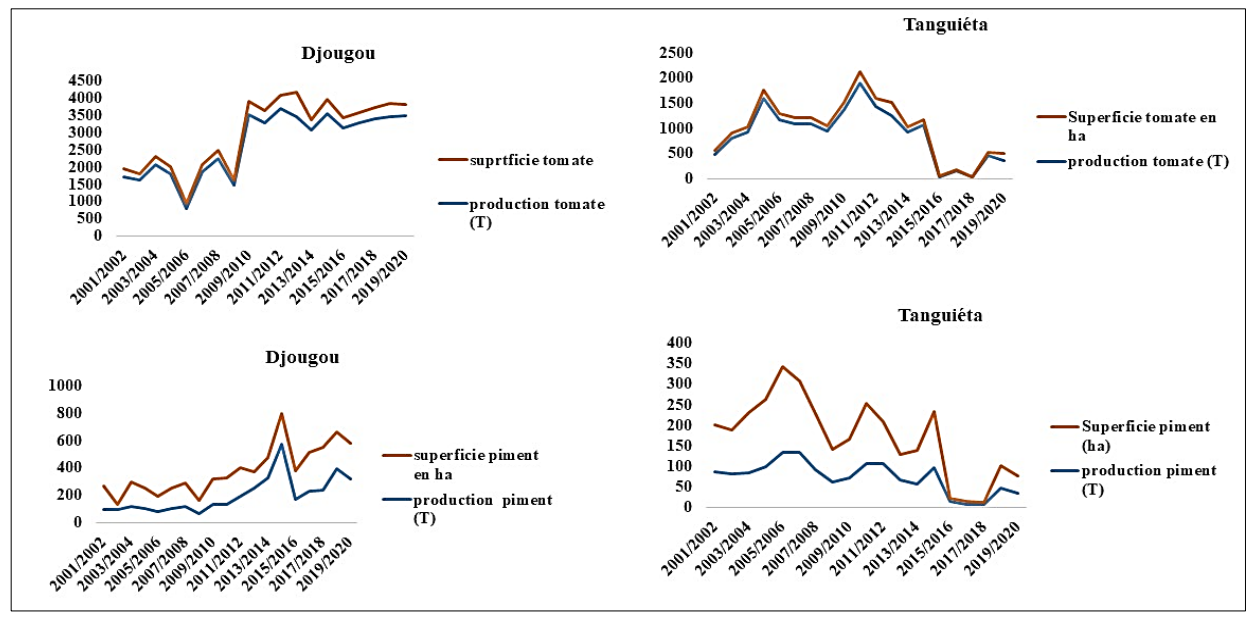

Figure 7 : Relation superficie et production.

Tableau 1 : Coefficients de corrélation de Spearman entre les superficies et les productions de 02 cultures.

\begin{tabular}{llcc}
\hline & Corrélation de Spearman & Djougou & Tanguieta \\
\cline { 2 - 4 } Tomate & Coefficient cor & 0,935 & 0,875 \\
& Spearman & 0,001 & 0,001 \\
\hline \multirow{2}{*}{ Piment } & Coefficient cor & 0,649 & 0,930 \\
\hline
\end{tabular}

Tableau 2 : Coefficients de corrélation de Spearman entre les rendements de 02 cultures et la pluviométrie.

\begin{tabular}{llcc}
\hline & Corrélation de Spearman & Djougou & Tanguieta \\
\cline { 2 - 4 } Tomate & Coefficient cor & 0,455 & 0,114 \\
& Spearman & 0,05 & 0,642 \\
\hline \multirow{3}{*}{ Piment } & Coefficient cor & 0,002 & 0,109 \\
\hline & Spearman & 0,994 & 0,26 \\
\hline
\end{tabular}

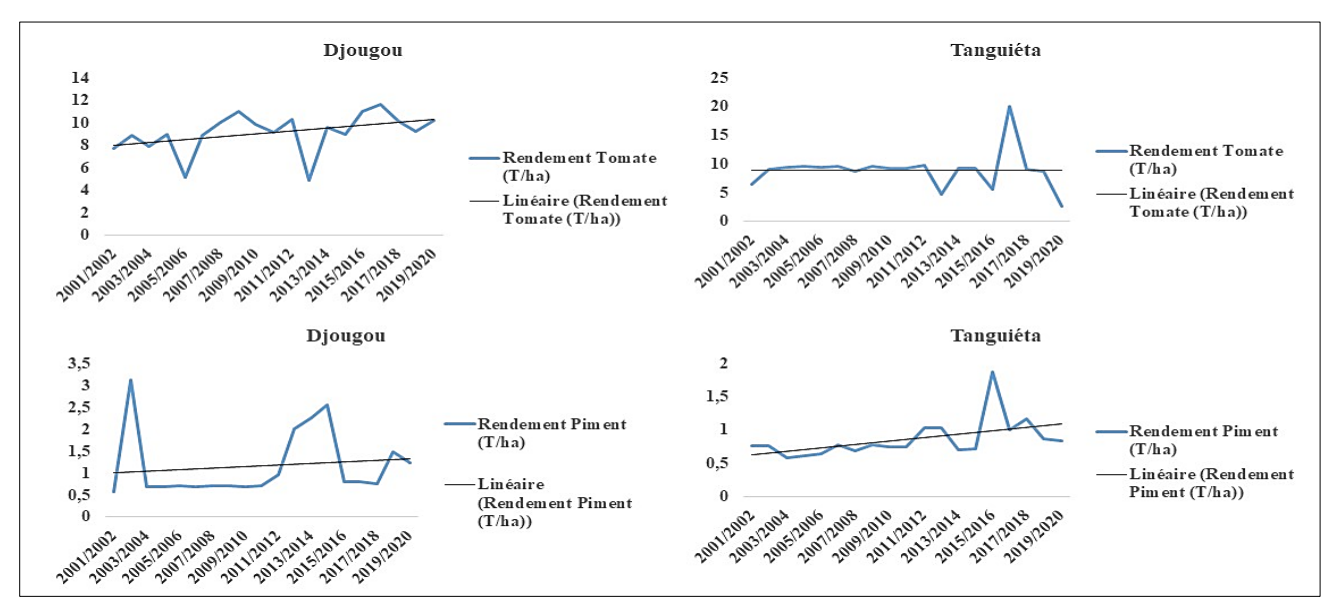

Figure 8 : Evolution des rendements. 


\section{DISCUSSION}

La mauvaise couverture des besoins en eau des cultures a été constatée dans la zone d'étude. Ces résultats corroborent ceux de Pige et al. (2001) et Houssou (2014) selon qui la petite saison sèche tend à disparaître, laissant la place à une longue saison des pluies dont l'installation est plus tardive.

Les tests de ruptures ont permis de détecter des années de rupture à Djougou en 1992 et à Tanguiéta en 1989. Cette variabilité climatique qui se caractérise par l'alternance d'années déficitaires et excédentaires est conforme à celle observée en Afrique de l'Ouest (Ardoin, 2004) et particulièrement au nord Bénin (Vissin, 2007 ; Vissin et al., 2007 ; Koumassi, 2014 ; Gbassi, 2018).

Par rapport à la température, une tendance à la hausse a été notée dans la zone de l'étude. Cette évolution est conforme à celle obtenue au sud du Bénin (Codjo et al., 2013) et au nord Bénin (Ogouwale 2006 ; Boko et al., 2012). Selon GIEC (2001), d'ici 2100 on assistera à l'élévation de la température de l'ordre de $5,8{ }^{\circ} \mathrm{C}$. La pression anthropique sur les essences végétales, les feux de végétation et la déforestation en sont les principales causes (Ouorou Barre, 2010)

Par rapport à la relation pluierendement, le test de corrélation de Spearman effectué montre une faible corrélation non significative à $95 \%$ entre la pluviométrie et les rendements. Ces résultats sont conformes à celles de certains auteurs. Selon les travaux de Ogouwalé (2006), les impacts des péjorations pluviométriques et de la hausse des températures sur les cultures se manifestent fréquemment par les stress hydriques et/ou thermiques. La seule connaissance du régime pluviométrique ne suffit pas à expliquer le rendement d'une culture ; il y a également l'état de la fertilité des sols, la qualité des semences, maladies et ravageurs qui jouent un rôle déterminant (Ouorou Barre, 2010 ; Noufé et al., 2011).

\section{Conclusion}

Cette étude indique clairement que le climat a connu une variation dans la zone de l'étude caractérisée par une succession des années déficitaires et des années excédentaires. Cette variabilité a été confirmée par le test de rupture de Pettitt (1979) qui s'est révélée significative au seuil de $95 \%$.

Cette variation climatique a eu un faible impact sur les superficies emblavées et rendements maraichers. En effet, les superficies emblavées et les rendements de tomate et de piment ont continué à croitre tant en années déficitaires qu'excédentaires. Cette faible corrélation a été confirmée par le test de Spearman au seuil de significativité de $95 \%$. Ce qui montre clairement que les modifications des conditions climatiques ne suffisent pas à expliquer la dynamique d'ensemble du développement végétatif de la tomate et du piment et ne saurait constituer un facteur limitatif majeur pour leur production. Dans ce contexte d'incertitude, les producteurs en fonction de leur perception de la variabilité climatique développent des techniques adaptatives dont l'analyse détaillée sera une priorité pour une autre étude.

\section{CONFLIT D'INTERETS}

Les auteurs déclarent qu'ils n'ont pas de conflit d'intérêts lié à cet article.

\section{CONTRIBUTIONS DES AUTEURS}

MBD, auteur principal de l'article a participé à toutes les étapes du travail. BOW a contribué à la collecte et à l'analyse des données. AJY, superviseur du travail a procédé à la correction de tout le document.

\section{REMERCIEMENTS}

Les auteurs remercient le Directeur de Programmation et de la Prospective du Ministère de l'Agriculture de l'Élevage et de la Pêche (MAEP) et les Responsables de l'Agence pour la Sécurité de la Navigation Aérienne en Afrique et à Madagascar 
(ASECNA) pour la qualité des données partagées.

\section{REFERENCES}

Ardoin BS. 2004. Variabilité hydro climatique et impact sur les ressources en eau de grands bassins hydrographiques en zone soudano-sahélienne. Thèse de Doctorat de l'Université de Montpellier II (France), $330 \mathrm{p}$.

Atidegla CS. 2011. Effets des différentes doses d'engrais minéraux et de la fiente de volaille sur l'accumulation de biocontaminants et polluants (germes fécaux, composés azotés et phosphorés, métaux lourds) dans les eaux, les sols et les légumes de Grand-Popo au Bénin. Thèse de doctorat unique, EDP/FLASH, Université d'Abomey-Calavi (UAC), Bénin, p 319.

Boko M, Kosmowski F, Vissin WE. 2012. Les Enjeux du Changement Climatique au Bénin. Programme pour le Dialogue Politique en Afrique de l'Ouest. KonradAdenauer-Stiftung, Cotonou, 65 p. DOI: http://dx.doi.org/10.13140/RG.2.1.2825. 4808.

Cocheme J, Franquin P. 1969. Une étude d'agro climatologie de l'Afrique sèche au Sud du Sahara en Afrique Occidentale, $p$ 87.

https://www.documentation.ird.fr/hor/fdi :11613.

Codjo HT, Lamodi F, Agbelessi S, Ogouwale R, Ogouwale E. 2013. Stratégies paysannes d'adaptation aux changements climatiques dans la Commune de Pobè. Actes du $26^{\text {ème }}$ Colloque de l'Association Internationale de Climatologie, Cotonou, 164-16.

Djohy GL. 2015. Variation climatique et production vivrière : la culture du maïs dans le système agricole péri-urbain de la commune de Parakou au Nord-Benin. Afrique SCIENCE, 11: 183-194. https://hal.archives-ouvertes.fr/hal01540739.

Doukpolo B. 2014. Changements climatiques et productions agricoles dans l'Ouest de la République Centrafricaine. Thèse de troisième cycle, Université d'AbomeyCalavi (UAC), République du Bénin, PDF, 338p.

Eldin M. 1989. Analyse et prise en compte des risques climatiques pour la production végétale. Le risque en agriculture, Dynamique des systèmes agraires, Orstom, collection à travers champs, Paris, $47-63$. DOI : https://doi.org/10.4000/books.irdeditions .16092

FAOSTAT. 2020. Répartition de la superficie ensemencée et de la production des cultures primaires. Divisions Statistiques de la FAO, www.countrystat.org (consulté le 25/01/2021).

Faye C. 2017. Une évaluation comparative des séquences de sécheresse météorologique par indices, par échelles de temps et par domaines climatiques au Sénégal. J. Wat. Environnement Science, 1(1) : 11-28.

Gbassi A. 2018. Pratiques agricoles d'adaptation aux aléas climatiques et performance économique des producteurs de Maïs dans les localités rurales du Nord-Est Bénin. Thèse de doctorat de l'EDSAE-UP.

GIEC. 2001. Pauvreté et changements climatiques. Rapport sur Réduire la vulnérabilité des populations pauvres par l'adaptation aux changements climatiques. Berlin Media Company, Allemagne, 43p.

GIEC. 2007. Bilan 2007 des changements climatiques. Contribution des Groupes de travail I, II et III au quatrième rapport d'évaluation du GIEC. Genève, 103 p.

Houssou SE. 2014. Variabilité climatique et production maraîchère dans le bas-fond de Houinga-Houégbé (Commune de 
Houéyogbé). Mémoire de Master II, FSA/UAC, p 119.

INSAE (Institut National de la Statistique et de l'Analyse Economique). 2003. Troisième recensement général de la population et de l'habitation. Synthèse des résultats, $p$ 34.

Jouilil I, Bitar K, Salama H, Amraou I, Mokssit A, Tahiri M. 2013. Sécheresse météorologique au bassin hydraulique OUM ER RBIA durant les dernières décennies. Larhyss Journal, 12: 109-127. http://larhyss.net/ojs/index.php/larhyss/ar ticle/view/151.

Koumassi DH. 2014. Risques hydroclimatiques et vulnérabilités des écosystèmes dans le bassin versant de la Sota à l'exutoire de Coubéri. Thèse de Doctorat Unique, EDP/FLASH, UAC, p 245.

McKee TB, Doesken NJ, Kleist J. 1993. The Relationship of Drought Frequency and Duration to Time Scales. Preprints, 8th Conference on Applied Climatology, Anaheim, 17-22 January 1993, 179-184.

Morel M, Braud R, Quantin P. 2008. Fluctuation climatique et variabilité interannuelle des cultures dans un essai d'assolement à long terme en Centrafrique. Bulletin de la Société Géographique de Liège, 51 : 139-149.

Morel R. 2004. Le climat et l'implantation des hommes : le cas de la Côte d'Ivoire. Annales A IC, 1 : 117-132.

Moron V. 1993. Variabilité des précipitations en Afrique tropicale au nord de l'équateur (1933-1990) et relations avec les températures de surface océanique et la dynamique de l'atmosphère. Thèse de doctorat. Centre de recherches de Climatologie, Université de Bourgogne, Dijon, 219 p. + atlas.

Noufé D, Mahé G, Kamagaté B, Servat E, Goula Bi Tié A, Savané I. 2015. Climate change impact on agricultural production: the case of Comoe River basin in Côte
d'Ivoire. Hydrologie Sci. J., 60(11): 1972-1983.

DOI : http://dx.doi.org/10.1080/02626667.2015 .1032293

Noufe D, Kouadio ZA, Soro GE, Wayou1 TP, Goula BTA, Savane I. 2015. Impact de la variabilité climatique sur la production du maïs et de l'igname en zones Centre et Nord de la Côte d'Ivoire. Agronomie Africaine, 27(3): 241-255. https://www.ajol.info/index.php/aga/artic le/view/130407.

Noufé D, Lidon B, Mahe G, Servat E, Brou YT, Koli BZ, Chaléard JL. 2011. Variabilité climatique et production de maïs en culture pluviale dans l'Est ivoirien. Hydrol. Sci. J., 56 (1) : 152 - 167. DOI :

https://doi.org/10.1080/02626667.2010.5 45247.

Ogouwalé E. 2006. Changements climatiques dans le Bénin méridional et central : Indicateurs, scénarios et prospective de la sécurité alimentaire. Thèse de Doctorat, UAC, FLASH, 302 p.

Ouorou Barre FI. 2010. Variabilité climatique et production agricole dans les communes de Tanguiéta et Matéri. Mémoire de DEA à UAC, Bénin, 109 p.

PANA. 2008. Convention-cadre des Nation Unies sur les changements climatiques. Programme d'Action National d'Adaptation aux changements climatiques du Bénin (PANA- Bénin), MEPN, Cotonou, Bénin81 p.

Pettitt AN. 1979. A Non-Parametric Approach to the Change-Point Problem. Journal of the Royal Statistical Society, 28(2): 126135.

Pige J, Biaou F, Vigan O. 2001. Zonage géographique et typologie de fonctionnement des exploitations agricoles. Projet d'amélioration et de diversification des systèmes d'exploitation (PADSE) Départements de l'Alibori, du Borgou, des Collines et du 
Zou. Synthèse des résultats des missions d'appui au volet enquêtes villageoises (octobre 2000-mai 2001). PADSE, Parakou, Bénin, p 64.

Reddy KR, Hodges RF. 2002. Climate Change and Global Crop Productivity. CABI Publishing: Wallingford.

Reyniers FN, Forest F. 1990. La pluie n'est pas le seul remède à la sécheresse en Afrique. Sécheresse, 1: 36-43. https://agritrop.cirad.fr/417113/

Salack S, Traoré BS, Sarr B. 2006. Synthèse sur la collecte, la mise en forme et le stockage des données climatologiques des pays du CILSS, et Etude d'impacts des changements climatiques sur la production agricole au Sahel, Rapport de stage, Centre Régional AGRHYMET, 95 p.

Sarr B. 2006. INSTAT+ en bref Manuel d'utilisation destiné aux Ingénieurs en agrométéorologie et en météorologie aéronautique, CILSS, Centre régional Agrhymet, $74 \mathrm{p}$.

SDSN. 2015. Sustainable Development Solutions Network, Getting Started with the Sustainable Development Goals. http://unsdsn.org/wpcontent/uploads/201 5/12/151211-getting-started-guideFINAL-PDF-.pdf .

Seck M, Moussa NA. 2005. Adaptation aux Changements Climatiques. L'étude de cas des systèmes de production agricoles de Sébikotane (Sénégal) Linking Climate Adaptation Project, 33 p.

Sossa B. 2001. Contribution à la mise en valeur de la plaine d'inondation du lac Toho à Kponou (S/P Athiémé, Département du Mono), Thèse d'Ingénieur Agronome, FSA/UNB, p 84.

Vissin E, Houndenou C, Perard J. 2007. Sécheresse pluviométrique et tarissement dans le bassin de la Mékrou (Bénin, Afrique de l'Ouest). Actes du $10^{\text {ème }}$ Colloque de AIC, Cathage Tunis, 568 573.

Vissin EW. 2007. Impact de la variabilité et de la dynamique des états de surface du bassin versant du fleuve Niger. Thèse de doctorat, Université de Bourgogne, CRC, Dijon, p 310. 